\title{
Akupunktur zur Migräne-Prophylaxe
}

\section{Eine deutsch-chinesische Studie hat die Wirksamkeit der Akupunktur in der Migräne-Prophylaxe überprüft.}

- Die Autoren randomisierten $480 \mathrm{Mi}$ gränepatienten in vier Gruppen, denen entweder eine von zwei Formen der traditionellen chinesischen Akupunktur (Shaoyang und Yangming) oder die dazugehörigen jeweiligen Scheinbehandlungen (Placebo) verabreicht wurden.

Der primäre Studien-Endpunkt war die Anzahl der Tage mit Migräneattacken in dem Zeitraum von fünf bis acht Wochen nach Studienbeginn. Die Auswertungen zeigten keine signifikanten Differenzen dieses Parameters. Dennoch wurde von den Autoren der Schluss gezogen, dass die Akupunktur klinisch positive Effekte verursachte.

- Y. Li et al.

Acupuncture for migraine prophylaxis: a randomized controlled trial. CMAJ 2011; DOI.10.1503.cmaj.110551

\section{Kommentar}

Diese Studie gibt mir Rätsel auf. Wie kann es sein, dass ein negatives Resultat stillschweigend in das Gegenteil umgekehrt wird? Zur Ehrenrettung der Autoren ist zu bemerken, dass einige sekundäre Endpunkte tatsächlich ein signifikant positives Ergebnis aufzeigen. Allerdings ist hier die Effektgröße gering, sodass die Autoren dies als nicht klinisch relevant bezeichnen. Auch eine Post-
hoc-Analyse von gepoolten Gruppen liefert ein positives Resultat. Solche Daten können interessant sein, sie sind jedoch nicht ausschlaggebend. Ist es also legitim, aus einer letztlich negativen Studie positive Schlüsse abzuleiten? Die meisten Experten würden hier wohl mit nein antworten. Dennoch passiert dergleichen im Bereich der Alternativmedizin leider viel zu häufig.

E. ERNST =

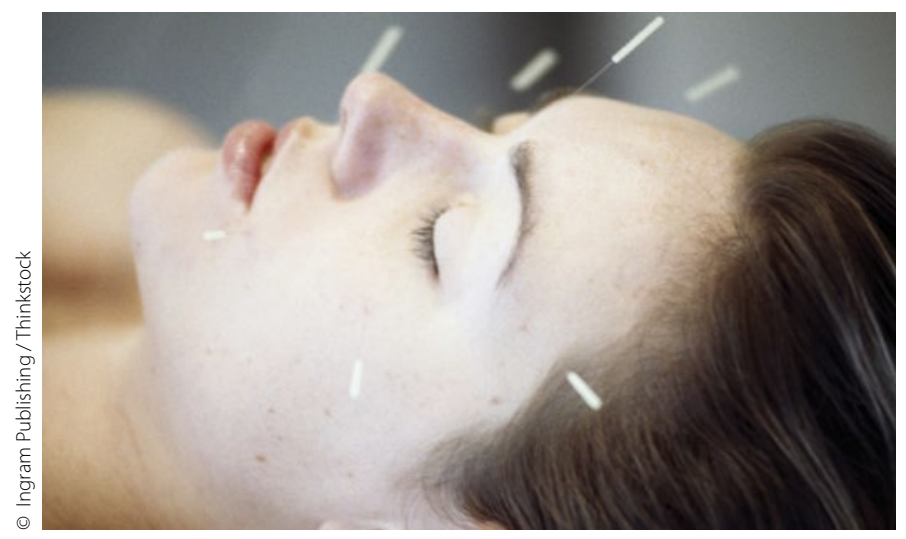

Was bewirkt

Akupunktur bei Kopfschmerzen?

\section{Überstunden erhöhen das Depressionsrisiko}

Die Beziehungen zwischen Mehrarbeit und einer Depression wurde jetzt von Wissenschaftlern der Universität in Helsinki und der Universität London überprüft.

- In einer prospektiven Kohortenstudie flossen Daten von 1626 Männern und 497 Frauen ein. Im Mittel waren sie 47 Jahre alt. Sie wurden zwischen 3,8 und 7,2 Jahre begleitet. $11 \%$ von ihnen arbeiteten im Schnitt elf Stunden und mehr am Tag. In einer ersten Untersuchung wurde ausgeschlossen, dass die Probanden bereits psychische Beschwerden hatten.

Insgesamt entwickelten 66 der 2123 Befragten (3,1\%) eine schwere Depression. Von den 1105 Menschen mit sieben bis acht Arbeitsstunden waren es 38, bei den 227 Befragten mit elf bis zwölf Stunden zehn Betroffene. Aus diesen Zahlen rechneten die Forscher anschließend

den Einfluss anderer Faktoren heraus und kamen auf eine OR von 2,43 (95\%$\mathrm{KI}=1,11-5,30)$. Demnach war die Wahrscheinlichkeit für eine schwere Depression für die Vielarbeiter doppelt so hoch wie für Angestellte, die keine Überstunden leisteten.

\section{Kommentar}

Überstunden prädisponieren zu schweren depressiven Phasen. Auch wenn Überstunden Vorteile für den Einzelnen und die Gesellschaft haben können, ist es wichtig zu wissen, dass übermäßige Arbeitsstunden auch mit einem höheren Risiko für schwere Depressionen einhergehen. Die Autoren schränken ein, dass sie keine plausiblen Erklärungen für das vermehrte Auftreten von Depressionen für Menschen mit vielen Überstunden haben. Aus anderen Studien gibt es Hinweise, dass lange Arbeitszeiten
- M. Virtanen et al.

(Finnish Institute of Occupational Health, Helsinki, Finland; E-mail: marianna.virtanen@ttl.fi) Overtime work as a predictor of major depressive episode: A 5-Year follow-up of the whitehall II study. Published online on 25 January 2012 in PLoS ONE 7(1): e30719. doi:10.1371/ journal.pone.0030719 zu Familienkonflikten, weniger Entspannung zwischen den Arbeitstagen und mehr Stresshormonen im Blut führen können. Es gibt aber auch Untersuchungen, die keinen Zusammenhang zwischen langen Arbeitszeiten und Depressionen gefunden haben. Die Probanden der vorliegenden Studie wurden aus im öffentlichen Dienst Beschäftigten rekrutiert. Weitere Studien beispielsweise mit Fabrikarbeitern oder im privaten Sektor sind daher nötig, um die Allgemeingültigkeit der Ergebnisse zu überprüfen. K. MALBERG = 